\title{
Research on the feature extraction of DC space charge behavior of oil-paper insulation
}

\author{
TANG Chao $^{1,2^{*}}$, LIAO RuiJin $^{2}$, CHEN George ${ }^{3} \&$ YANG LiJun $^{2}$ \\ ${ }^{1}$ College of Engineering and Technology, Southwest University, Chongqing 400715, China; \\ ${ }^{2}$ The State Key Laboratory of Power Transmission Equipment \& System Security and New Technology, \\ Chongqing University, Chongqing 400044, China; \\ ${ }^{3}$ School of Electronics and Computer Science, University of Southampton, Southampton SO17 3BJ, UK
}

Received September 3, 2010; accepted December 13, 2010; published online January 25, 2011

\begin{abstract}
As the ultra high DC voltage grid comes into an important stage in the 'Twelfth Five-year Plan' of China, the requirement on the operation safety of power equipment needs to be improved. Oil-paper insulation plays a significant role as the main insulation material of many key power equipments. The space charge behaviors of oil-paper insulation not only affect the dielectric strength, but also relate to the stability and security of power transmission facilities. Until now, the research on the space charge behaviors of oil-paper insulation remains on the stage of objective description and phenomenon explanation, thus it is very urgent to make some feature extractions on the test results in order to further investigate the DC space charge behaviors of oil-paper insulation. In this paper, based on the large numbers of test data in the first stage, data mining and feature extractions were performed on the DC space charge behavior of oil-paper insulation. The variation laws of some key characteristic parameters obtained in different experiment conditions such as the total charge injection amount, apparent charge mobility, and trap energy distribution were analyzed. In addition, the distribution of trapping level inside oil-paper was calculated by using the PEA test results based on the mechanism of the isothermal decay current, which realized the approximate calculation of trap depth by charge decay characteristics. What's more, the exponential injection and decay equations of DC space charge changing with time were acquired for the first time, and the physical meanings of related parameters were defined. This has laid a foundation for the prediction and simulation of space charge in the oil-paper insulation.
\end{abstract}

\section{oil-paper insulation, space charge, DC, feature extraction}

Citation: Tang C, Liao R J, Chen G, et al. Research on the feature extraction of DC space charge behavior of oil-paper insulation. Sci China Tech Sci, 2011, 54: 1315-1324, doi: 10.1007/s11431-010-4268-x

\section{Introduction}

In China, the fast expanding HVDC networks require higher secured and stable DC power equipment. As the main insulation material of some key DC power equipment, oil-paper is vital important for the operation security. The space charge behaviors affect the dielectric strength of oil-paper, cause the formation of discharge channels and damage its

*Corresponding author (email: tangchao_1981@163.com) insulating properties, which made the research on space charge behaviors of oil-paper quite significant for the operation security of power grid, as well as the improvement on the physical chemical properties and electric performances of insulation.

It is generally approved that because of the electric field distortion effect, space charge inside insulation materials affects the conductance, breakdown voltage, and ageing process evidently [1]. In 1970s, Tanaka and Tanaka in Japan researched a lot on the space charge behavior [2-6]. Mazzanti and Montanari in Italy focused on the space 
charge characteristics of polyethylene materials, and studied the threshold voltage and apparent charge mobility [7-9]. In China, Tu and Zhang started the research on the space charge test methods and equipment using the pulsed electroacoustic (PEA) technique in the late 1980s [10-16]. Thereafter, Lei [17], Zhou [18-20] et al. performed nvestigations on the space charge measurement techniques.

As for the oil-immersed insulation paper (pressboard), the pressure wave propagation (PWP) method was used for the first time to measure the space charge and the electric field distributions under DC electric field in 1994 [21, 22]. Due to the hygroscopic nature, the charge measurements in an oil-impregnated pressboard are much more complicated than that in polymers, and the tests results were not very satisfactory at that time. From 1997, a few studies were performed on oil-paper insulation using PEA. Morshuis and Jeroense made some space charge tests on oil impregnated insulation paper, and it was the first time for results on space charge measurements on HVDC cable paper insulation to be published [23, 24]. Ciobanu et al. analyzed the space charge evolution in oil-paper insulation, and associated the mobility phenomena with the mineral content of cable paper $[25,26]$.

Recent researches in China reported some interesting information about space charge behaviors in oil-paper insulation. Zhou discussed the relationship between space charge and breakdown in oil-paper. The results showed that the space charge came from ionization at lower field intensity [27]. Besides, space charge behaviors in the multi-layer oil-paper insulation system were analyzed and the influence of temperature on charge dynamics was discussed in our prior research works [28-30].

In this paper, based on a large number of previous research results obtained by PEA methods, data mining and feature extractions were made on the DC space charge behavior of oil-paper insulation. The variation laws of some key characteristic parameters obtained under different experiment conditions such as the total charge injection amount, apparent charge mobility, and trap energy distribution were analyzed. In addition, the distribution of trapping level inside oil-paper was calculated by using the PEA test results based on the mechanism of the isothermal decay current, which realized the approximate calculation of trap depth by charge decay characteristics. What's more, the exponential injection (decay) equations of DC space charge changing with time were acquired for the first time, and the physical meanings of related parameters were defined, which laid a foundation for the prediction and simulation of space charge in the oil-paper insulation.

\section{Experiment samples and equipment}

The measurements in this paper were carried out on oil immersed insulation paper. The insulation paper made by
Croylek Ltd. has a thickness of $\sim 60 \mu \mathrm{m}$ for a single layer. The parameters of the paper meet the international standard of IEC 554.2. The insulation oil used in this experiment was Nytro 10X transformer oil, provided by Nynas Oil Company. Nytro $10 \mathrm{X}$ is an inhibited transformer oil with good electrical properties and oxidation stability, it has a wide industrial application as well.

The PEA system (PEANUT, made by Five Lab) used in this study has a pulse width of $5 \mathrm{~ns}$ and amplitude of 0-600 V. The bottom electrode is made of $10 \mathrm{~mm}$ thick aluminum plate and the top electrode is semiconducting polymer to achieve a better acoustic match. The piezoelectric sensor used was a $9 \mu \mathrm{m}$ thick $\mathrm{LiNbO}_{3}$ material.

Several pretreatment steps, the same as in ref. [30], were carried out before thermal ageing. After that, the space charge measurements were taken on three-layer samples at various times during the periods of both volts-on and voltsoff (short-circuit condition) using the PEA system. The test results and preliminary analysis were also shown in ref. [30].

\section{Total charge}

Under the stress of DC voltage, space charge injected into the dielectric materials, accumulated locally, resulted in the electrical field distortion and the damage of dielectrics. The total space charge that related to the electric performance as well as the physical, chemical and microcosmic characteristics represents the property of space charge transport inside the material.

The total absolute amount of charge accumulated in the samples can be calculated based on the charge-density distribution by

$$
Q(T)=\int_{0}^{d}|\rho(x, t)| S \mathrm{~d} x
$$

where $\rho(x, t)$ is the charge density; $S$ is the electrode area; $d$ is the thickness of the sample.

As we know, there is always a trend of movement and combination exists between positive and negative charges. So the total charge is always changing during volts-on and volts-off tests. In this paper, the total charge at the time immediately after power on (off) in the volts-on (volts-off) test was calculated.

\subsection{Different applied DC voltages}

At the normal temperature $\left(20^{\circ} \mathrm{C}\right)$, the evolution of total space charge during the whole volts-on experiment was shown in Figure 1. When the applied DC voltage was $4 \mathrm{kV}$, the total charge inside oil-paper sample increased with the increase of DC stressing time and then tended to a stable state. Under $6 \mathrm{kV}$, the total charge decreased a little at prophase and then tended to be stable. The same happened under $8 \mathrm{kV}$, the total charge became stable after small fluctuation. 


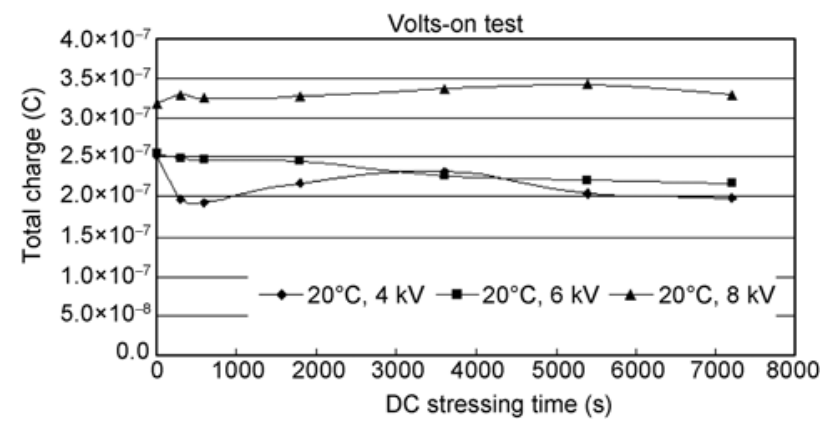

Figure 1 Total charges under different applied DC voltages vs. time (Volts-on at $20^{\circ} \mathrm{C}$ ).

The volts-on measurement $Q(t)_{\text {on }}$ includes contributions from both fast and slow charges while the volts-off measurement $Q(t)_{\text {off }}$ (turn off the power, test the space charge dynamics, then turn on the power) involves slow charges mainly. The slow moving charges indicate the information of shallow traps inside oil-paper. Figure 2 shows that as for the un-aged oil-paper, the total fast moving charges doubled that of slow moving charges.

The total space charge distributions after the removal of the applied voltage (decay test) are shown in Figure 3. The dissipation of total charge represents an exponential decay curve. The total charge amount decreased very fast at first then slowed down. Subsequently, charge decayed more slowly and finally to the ultimate state.

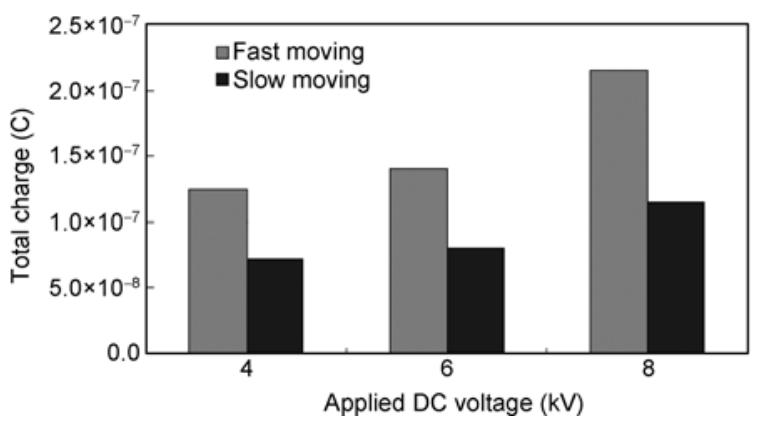

Figure 2 Total charges of fast moving and slow moving at the stable state under different applied DC voltages.

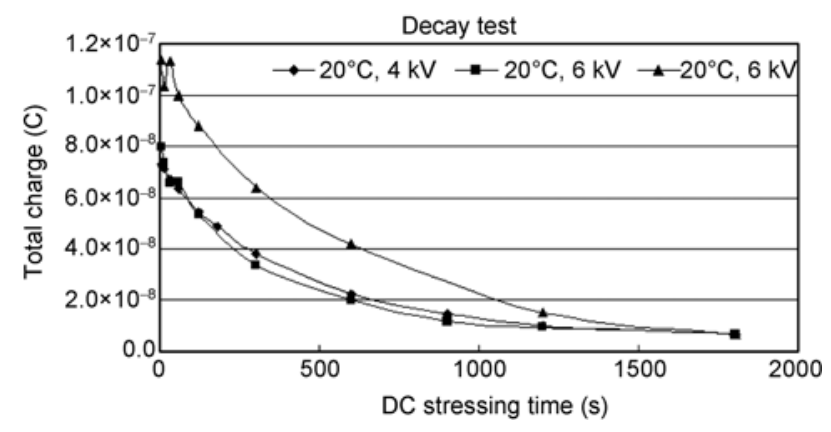

Figure 3 Total charge dynamics in decay test under different applied DC voltages $\left(20^{\circ} \mathrm{C}\right)$.

\subsection{Different temperatures}

\subsection{1 $40^{\circ} \mathrm{C}$}

When the test temperature increased to $40^{\circ} \mathrm{C}$, from the volts-on results as shown in Figure 4, it can be concluded that with the increase of DC stressing time $(4 \mathrm{kV})$, the total charge amount decreased at first then increased, finally tended to stable after 100 min DC stressing. Under $6 \mathrm{kV}$, similar to that at $20^{\circ} \mathrm{C}$, the total charge reached a stable state after a slight decrease, while under $8 \mathrm{kV}$, the total charge amount arrived at a stable state after fluctuation.

The total charge dynamics in decay test at $40^{\circ} \mathrm{C}$ (Figure 5) shows an exponential decrease, though there were a $20^{\circ} \mathrm{C}$ increase in temperature. In the beginning of decay test, because of the high residual electrical field, space charge inside oil-paper was de-trapped and neutralized very fast, which made the total charge decrease quickly. With the increase of decay time, on the one hand the effect of residual electrical field weakened, on the other hand, space charge remained inside oil-paper were mainly trapped in deep traps which requires more energy to de-trapping, therefore, the decay rate of total charge decreased continuously.

\subsection{2 $60^{\circ} \mathrm{C}$}

When the temperature increased to $60^{\circ} \mathrm{C}$, the total charge variations in volts-on test under different voltages were shown in Figure 6. Similar to those at $20^{\circ} \mathrm{C}$ and $40^{\circ} \mathrm{C}$, the total charge amount increased with the increase of DC voltage. Under different voltages, the total charge tended to

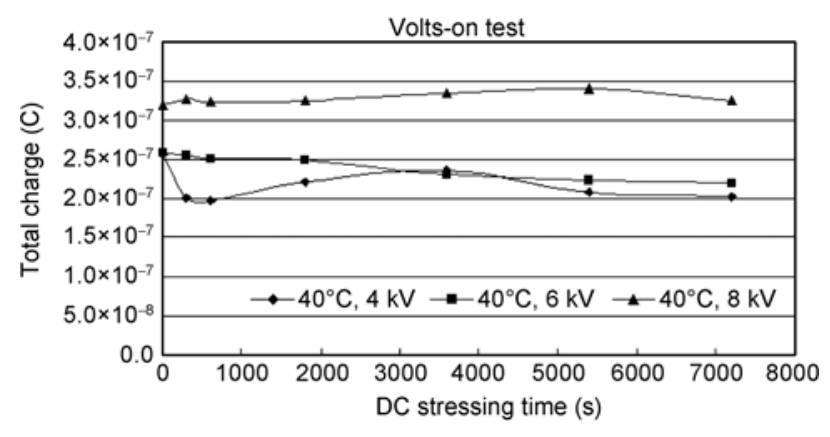

Figure 4 Total charges under different applied DC voltages vs. time (Volts-on at $40^{\circ} \mathrm{C}$ ).

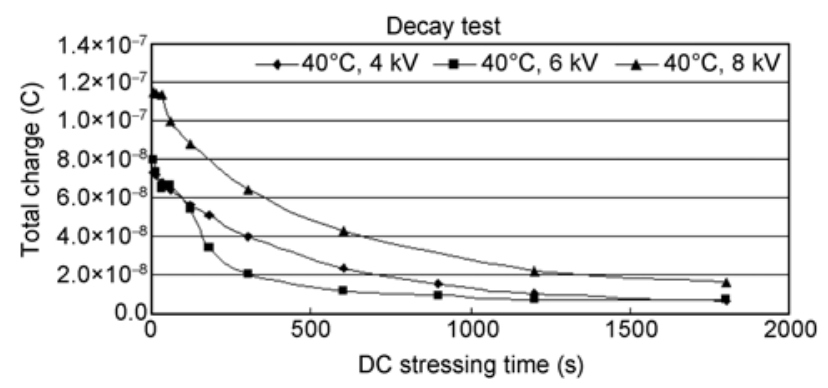

Figure 5 Total charge dynamics in decay test under different applied DC voltages $\left(40^{\circ} \mathrm{C}\right)$. 


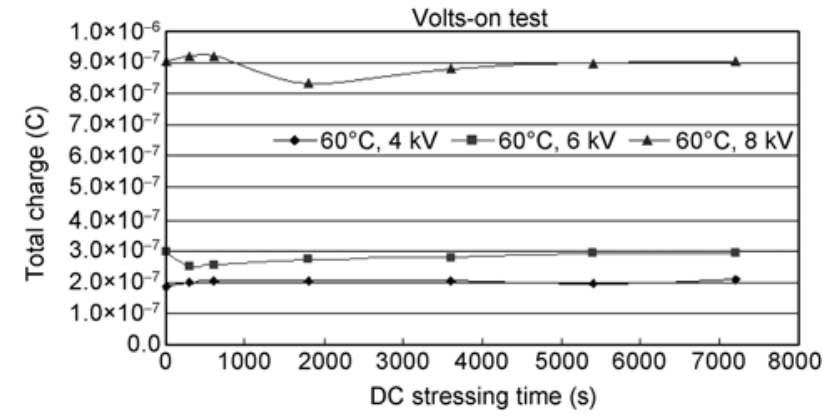

Figure 6 Total charges under different applied DC voltages vs. time (Volts-on at $60^{\circ} \mathrm{C}$ ).

a stable state after fluctuation.

Considering the space charge information at different temperatures and under different voltages, it is clear that under the extreme condition $\left(60^{\circ} \mathrm{C}, 8 \mathrm{kV}\right)$, namely high temperature and high voltage, both fast moving charge and slow moving charge increased largely, resulting in the increase of free carriers and strenuous vibration of electrons, and causing partial discharge and breakdown. Thus, special attention should be paid to the space charge behavior under high temperature and high voltage.

\section{Apparent charge mobility}

Space charge transition inside dielectrics includes polarization with electric power stress and depolarization without power. Generally, volts-on process is quite sensitive to the applied voltage grade and the surrounding environment. The volts-off process has a precise reflection of inside space charge movement and the characteristics of dielectrics itself. Therefore, the mobility of space charge in volts-off condition is a significant parameter for the research of space charge behavior. The space charge mobility $\mu$ can be calculated by

$$
\mu=v / E,
$$

where $v$ is the velocity of charge penetration into insulation from electrodes; $E$ is the mean electrical field.

The space charge mobility $\mu(t)$ can be obtained by

$$
\mu(t)=\frac{2 \varepsilon}{q(t)^{2}} \frac{\mathrm{d} q(t)}{\mathrm{d} t},
$$

where $q(t)$ is the charge density, is the dielectric permittivity, and $t$ is the depolarization time.

It is very important that the method above is to estimate the trap-controlled mobility using the depolarization characteristics, thus it is defined as 'apparent charge mobility'. Though different than the exact charge mobility, the advantage of using the method above is that it can easily calculate the charge mobility once the depolarization data is obtained, and acquire the de-trapping information, which is important for the research of dielectrics insulation performance.

\subsection{Different applied DC voltages}

At $20^{\circ} \mathrm{C}$, the apparent charge mobility under different applied voltages was shown in Figure 7. On the whole, the apparent charge mobility decreased first, and then increased to a maximum value, finally declined slowly. In the early short time (about $60 \mathrm{~s}$ ), there were some fluctuations, but the falling stage after the maximum value indicated the transmission information clearly. The higher the applied DC voltage was, the bigger the apparent charge mobility (max value) was. This is mainly due to the increase of accumulation charge inside oil-paper caused by the increase of applied voltage, and after the removal of applied voltage, a high residual electrical field resulted in a high charge mobility. After 300 s' decay, a trend of steady decrease was observed under all the three different voltages.

Corresponding to the decay curve of total charge amount, space charge inside oil-paper in volts-off test decreased gradually. The quantity of residual space charge lies in the depth and density of the charge traps; a large number of deep traps will lead to the charge accumulation. Meanwhile, the space charge decay curve indicates the apparent charge mobility; the larger the slope of the decay curve is, the bigger the apparent charge mobility is, and vice versa.

\subsection{Different temperatures}

\subsection{1 $40^{\circ} \mathrm{C}$}

At $40^{\circ} \mathrm{C}$, a rule of the apparent charge mobility after the removal of applied voltage similar to the rule at $20^{\circ} \mathrm{C}$ was observed in Figure 8. In the beginning (within $200 \mathrm{~s}$ ), the apparent charge mobility increased with fluctuations, and then decreased gradually after reaching the max value.

The variation of the apparent charge mobility with decay time is the reflection of de-trapping process of space charge captured by traps with different trap energies. However, the information of charge density acquired by the PEA reflects the charge carrier information of shallow traps only. Therefore, the charge trap discussed here is basically the shallow trap

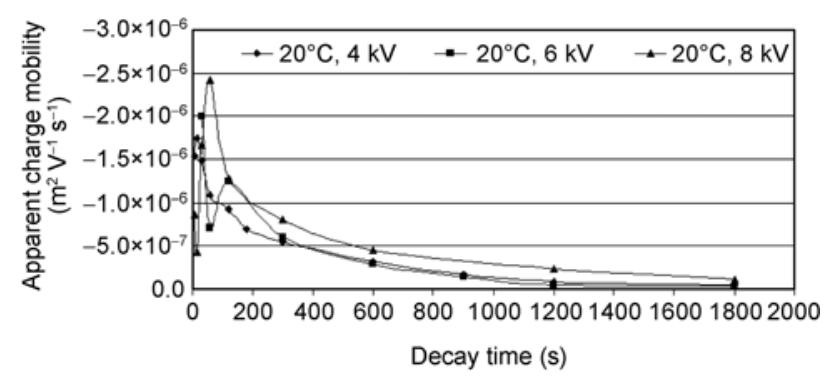

Figure 7 Apparent charge mobilities under different applied DC voltages vs. decay time $\left(20^{\circ} \mathrm{C}\right)$. 


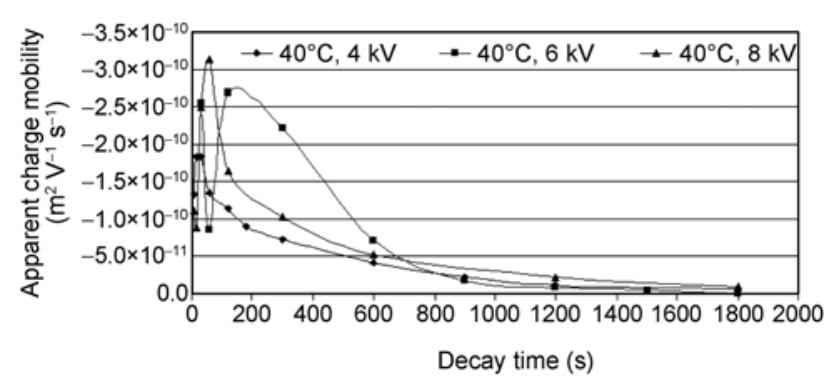

Figure 8 Apparent charge mobilities under different applied DC voltages vs. decay time $\left(40^{\circ} \mathrm{C}\right)$.

with a different trap depth.

As shown in Figure 8, the max value of the apparent charge mobility reduced obviously at $20^{\circ} \mathrm{C}$. It can be concluded that the relatively deeper trap increased when the temperature increased to $40^{\circ} \mathrm{C}$, thus the capability to restrain charge increased, and the apparent charge mobility decreased.

\subsection{2 $60^{\circ} \mathrm{C}$}

At $60^{\circ} \mathrm{C}$, the apparent charge mobilities under different applied DC voltages in decay tests were shown in Figure 9. It can be seen that the higher the applied voltage was, the bigger the apparent charge mobility after the removal of DC voltage was. And the variation of the apparent charge mobility showed a process of fast decrease at first and then tended to stable state. The fast decrease at first indicates the de-trapping of space charge inside oil-paper which restrained by the relatively shallow traps, while the slow decline reflects the de-trapping of space charge bounded in the relatively deep traps.

Under higher DC voltages in volts-on test at $60^{\circ} \mathrm{C}$, much more shallow traps were excited, more space charge accumulated inside oil-paper, which made the apparent charge mobility in the later stage increase with the increase of voltage applied in former volts-on tests.

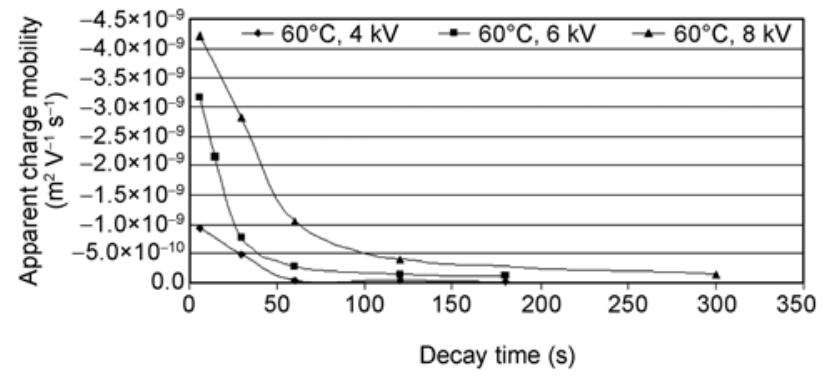

Figure 9 Apparent charge mobilities under different applied DC voltages vs. decay time $\left(60^{\circ} \mathrm{C}\right)$.

A combined analysis of the apparent charge mobility curves showed that the applied voltage had an effect on the trap exciting and formed new shallow traps, which increased the trap density. Or the applied DC voltage decreased the density of deep traps in a certain degree, which increased the apparent charge mobility under a high voltage.

As for the influence of temperature on the apparent charge mobility of oil-paper, the apparent charge mobilities at different temperatures under $4 \mathrm{kV}$ were shown in Figure 10. It is clearly that the apparent charge mobility at $20^{\circ} \mathrm{C}$ was much larger than those at $40^{\circ} \mathrm{C}$ and $60^{\circ} \mathrm{C}$. Cosidering the mechanism of charge trap formation inside the dielectrics, the increase in temperature excites some more the deep traps, which makes the density of deep traps increase and decreases the apparent charge mobility.

Therefore, the effect and the mechanism of temperature and applied voltage on the apparent charge mobility respectively are different. At the same temperature, a high applied DC voltage will reduce the depth of charge trap, and lead to the acceleration of the apparent charge mobility. On the other side, the increase in temperature will excite the formation of relatively deep trap inside oil-paper, strengthen the capability of bounding charge carrier, and result in a slow apparent charge mobility.

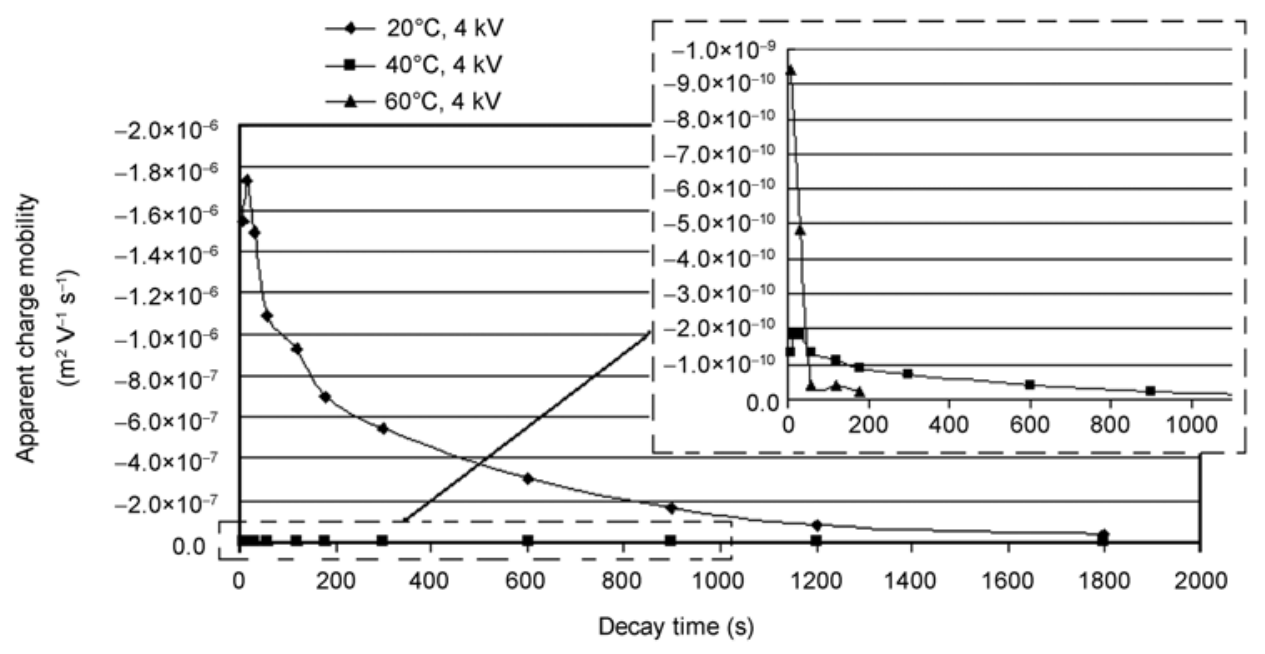

Figure 10 Apparent charge mobilities vs. decay time at different temperatures under $4 \mathrm{kV}$. 
It is noteworthy that from the present test results, there are some fluctuations in the effect made by the temperature and applied voltage on the apparent charge mobility. In order to acquire the detailed mechanisms, the measuring range has to be broaden, especially the temperature range.

\section{Trap energy distribution}

It is generally accepted by the researchers that the behavior and the distribution of space charge reflect the trap energy distribution. As for the research on the trap energy of dielectrics, the thermally stimulated current method (TSC) and space charge distribution measurement method are widely used to acquire the space charge information and study the dielectric performance from the microscopic view. In recent years, some researchers attempted to obtain the trap energy information by PEA method. Wang used PEA method to measure the decay property of negative charges after the specimen was short-circuited directly. Then energy distribution of surface trap was obtained through formula derivation and calculation [31].

In this paper, based on the research results at home and abroad, according to the isothermal charge equation, a method of studying the trap energy of oil-paper by using PEA test result was introduced.

The research indicates that the charge density has correlation with the current, and generally, charge condition inside dielectrics can be represented according to the measurement of surface potential. The average charge centroid after corona charging locates on the thin layer traps which is $\delta$ apart from the near surface, and the equivalent surface charge density $\sigma$ has a direct proportional relation with the surface potential $V_{S}$ [32], thus the surface potential can be calculated by

$$
\sigma=\varepsilon_{0} \varepsilon V_{S} / r^{\prime}
$$

where $\sigma$ is the equivalent surface charge density; $\varepsilon_{0}$ is the dielectric constant under vacuum; $\varepsilon$ is the relative dielectric constant; $V_{S}$ is the surface potential; $r^{\prime}$ is the average charge centroid, $120 \mathrm{pm}$.

The current density can be obtained by

$$
j=\frac{\varepsilon_{0} \varepsilon}{L} \frac{\mathrm{d} V_{S}(t)}{\mathrm{d} t}=\frac{r^{\prime}}{L} \frac{\mathrm{d} \sigma(t)}{\mathrm{d} t},
$$

because the relationship between the attenuation of surface potential and the current is

$$
i(t)=C \frac{\mathrm{d} V_{S}(t)}{\mathrm{d} t}
$$

where $C=\varepsilon_{0} \varepsilon A / L ; A$ is the surface area; $L$ is the thickness of test sample.

It is obvious that the current density is proportional to the reciprocal of surface potential and the reciprocal of surface charge density as well.

In the corona discharge, the variation of charge density in the trap (potential decay) is approximate to an exponential decay [33], which is

$$
\sigma=A \cdot \mathrm{e}^{-t / \tau},
$$

where $A$ is a constant; $\tau$ is the constant of decay time.

The variation law of the current density including the constant of decay time can be obtained by

$$
|j(t)|=\frac{r^{\prime}}{L} \frac{A}{\tau} \mathrm{e}^{-t / \tau} .
$$

As for oil-paper insulation material, when the applied voltage is removed, the carriers bounded in shallow traps are released firstly, and later the carriers bounded in deep traps are released. The release current which changes with decay time at different temperatures illuminates the distribution of trap energy in the sample surface. On the assumption that the carriers will not be trapped again, the relationship among the trap energy level $E_{t}$, the current density $j$ and the trap density $N_{t}$ can be calculated by

$$
\begin{gathered}
E_{t}=k T \ln v t, \\
j=\frac{q L k T}{2 t} f_{0}\left(E_{t}\right) N\left(E_{t}\right),
\end{gathered}
$$

where $f_{0}\left(E_{t}\right)$ represents the original occupation rate of traps inside the dielectrics, $1 / 2 ; q$ is the electron charge, $1.6 \times 10^{-19} \mathrm{C}$; $k$ is the Boltzmann's constant, $8.568 \times 10^{-5} \mathrm{eV} / \mathrm{K} ; T$ is the absolute temperature, $\mathrm{K} ; v$ is frequency of electron vibration, $3 \times 10^{12} \mathrm{~s}^{-1}$. The trap energy of electron is calculated from the bottom of the conduction band, and the trap energy of hole is calculated from the peak of the valence band.

$$
\text { If } \eta_{1}=\frac{q L k T}{2 t} f_{0}\left(E_{t}\right), \eta_{2}=\frac{r^{\prime} A}{L}, \eta_{1} \text { and } \eta_{2} \text { are constant, }
$$
then

$$
N\left(E_{t}\right)=\frac{\eta_{2}}{\eta_{1}} \frac{t}{\tau} \mathrm{e}^{-t / \tau}
$$

Eq. (11) indicates that the density of trap energy level $N\left(E_{t}\right)$ is connected closely with both the decay constant of charge density and decay time. Therefore, after obtaining the decay curve of surface charge by PEA data, with the decay time being constant, the surface trap energy distribution can be calculated by eq. (11).

\subsection{Different applied DC voltages}

Figure 11 shows the trap energy distribution of oil-paper under different voltages at $20^{\circ} \mathrm{C}$. The division of shallow and deep traps is not the same in different research areas. In the research domain of dielectrics, a shallow trap is defined 


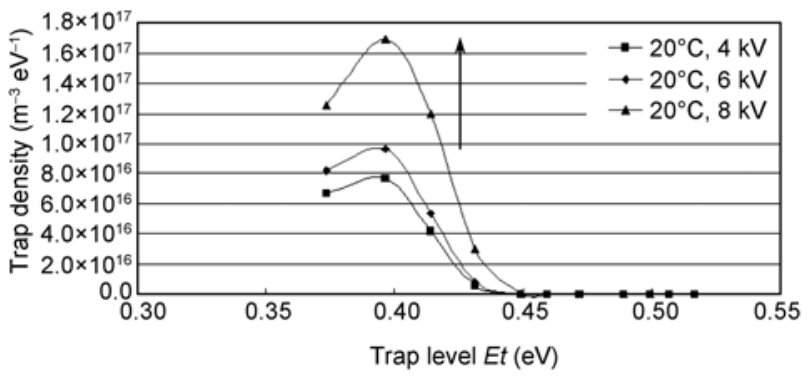

Figure 11 Trap energy distributions of oil-paper under different voltages at $20^{\circ} \mathrm{C}$.

under $1.10 \mathrm{eV}$, a deep trap is more than $1.10 \mathrm{eV}$. As shown in Figure 11, trap energy of oil-paper at $20^{\circ} \mathrm{C}$ was in the range of 0.38 to $0.58 \mathrm{eV}$. To get the information of lower trap energy, according to the theory of isothermal charge, very short-time space charge behavior has to be tested. For example, in order to get the information about trap of $0.30 \mathrm{eV}$, the isothermal charge decay within $10^{-5} \mathrm{~s}$ has to be measured, which is impossible for PEA equipment. But it is easy to obtain the relatively deep trap energy distribution by PEA and the surface potential technique.

It is noteworthy that the trap information shown here was reflected by space charge inside oil-paper which came from PEA data. The trap energy distribution in the surface and layer interfaces can be calculated once the charge decay in the surface or layer interfaces are obtained.

In Figure 11, the trap energy level was mainly in the range of 0.37 to $0.45 \mathrm{eV}$, the trap density increased at first then decreased, the highest trap density was in $0.4 \mathrm{eV}$, and very few trap energies were bigger than $0.45 \mathrm{eV}$.

On the basis of the trap theory [34], it can be seen from the comparison of trap energy distributions under different applied DC voltages that with the increase of voltage, the distribution curve tended upward, indicating that the trap density became larger because of the voltage excitation. Under the pressure of high voltage, the electrons from the cathode were injected into the conduction band, and then bounded by the trap quickly after a few times of scattering. In the process of charge trapping and recombination, the electrons transmitted from high energy state to low energy state, and the redundant energy was passed to another electron in a non-thermal radiation way to make it a hot electron [33]. When the hole injection happened, the energy released by hole trap passed to another electron in a non-thermal radiation way and formed a hot electron. As the formation and energy of hot electron are decided by the trap density and depth, altering the trap density and depth is a good way to change the probability and energy of hot electron formation, which is the foundation of improving the dielectric performance by material modification.

Therefore, the increase of applied voltage made the shallow trap density inside oil-paper increase, which increased the probability and energy of hot electron formation, accel- erated the damage to the cellulose chain, and led to the decline of chemical and physical performances and shorten the insulation life time.

\subsection{Different temperatures}

\subsection{1 $40^{\circ} \mathrm{C}$}

When the temperature increased to $40^{\circ} \mathrm{C}$, the trap energy distribution inside oil-paper was shown in Figure 12. The trap energy level was mainly within the range of 0.40 to $0.48 \mathrm{eV}$, and a similar change process with that at $20^{\circ} \mathrm{C}$ is observed. But the maximum value of trap depth moved to the trap level of $0.42 \mathrm{eV}$, and trap level higher than $0.48 \mathrm{eV}$ can be neglected because of the extremely low trap density.

\subsection{2 $60^{\circ} \mathrm{C}$}

When the temperature increased to $60^{\circ} \mathrm{C}$, as the charge decay rate was quite fast, three representative trap level distributions at different temperatures under $8 \mathrm{kV}$ were taken out to compare (Figure 13). The trap energy level obtained by charge density decay of oil-paper was within the range of 0.42 to $0.58 \mathrm{eV}$, and the maximum value of trap depth moved to the trap level of $0.51 \mathrm{eV}$, both of them were greater than those at $20^{\circ} \mathrm{C}$ and $40^{\circ} \mathrm{C}$. However, as mentioned before, the trap energy distribution acquired by PEA method was basically the shallow trap information (less than $1.10 \mathrm{eV}$ ).

As shown in Figure 13, the trap energy distribution changed with the increase of test temperature, and the

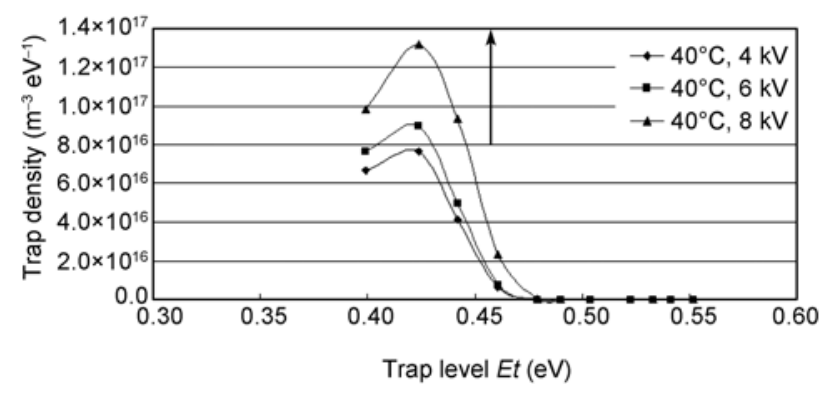

Figure 12 Trap energy distributions of oil-paper under different voltages at $40^{\circ} \mathrm{C}$.

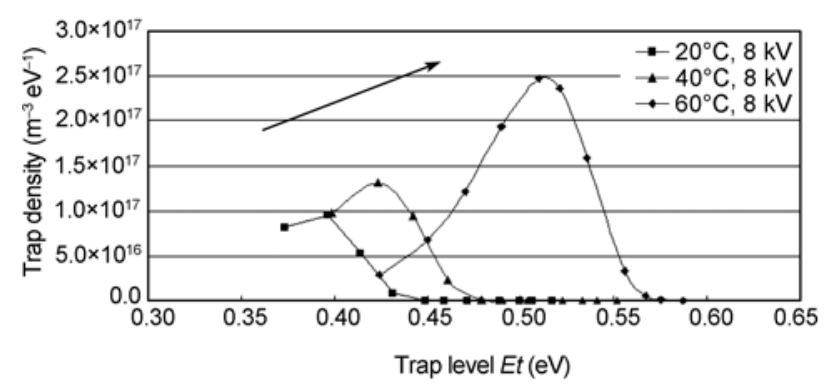

Figure 13 Trap energy distributions of oil-paper at different temperature under $8 \mathrm{kV}$. 
distribution curve shifted right upward. On the one hand, relatively deep traps were excited constantly, deeper traps formed with the increase of temperature. On the other hand, the trap density increased as well, especially at $60^{\circ} \mathrm{C}$.

Thus it can be concluded that under the same applied voltage, with the increase of temperature, the trap depth of oil-paper increased, as well as the density of relatively deep trap. As the tested trap was a shallow trap, the increase in the density of shallow trap increased the probability and energy of hot electron formation inside oil-paper, which enhanced the damage to the cellulose chain caused by the electron movement. In addition, a large number of shallow traps have great influence on the partial discharge behavior, deteriorate the chemical physical properties and shorten the insulation life time.

The analysis above established the relationship between depolarization and trap energy distribution, which realized the approximate calculation of trap energy in oil-paper through the decay test of space charge, though the calculation result reflects the shallow trap only and a lot of improvements are required.

\section{Charge injection and decay patterns}

As for the oil-paper insulation materials, homocharge injection occurs under DC stressing, space charge is injected from both electrodes and accumulated in the bulk. Afterwards, space charge is transferred, collided, and neutralized inside oil-paper sample, which makes the total charge amount changed accordingly.

After the removal of DC stressing, charge decay of the injected and accumulated space charge starts. It can be seen from the charge decay curves that the forms of charge decay is basically that the charge density in the position of charge accumulation decreases gradually, rather than the movement of a whole space charge package.
In general, relatively accordant increase and decrease patterns of total space charge, i.e., the injection and decay patterns, were observed in the tests. Especially, the charge decay process (Figures 3 and 5), after fitting by the exponential equation, had the goodness of fittest higher than 0.95 under all the test conditions. Therefore, the space charge decay inside oil-paper insulation obeys the law of exponential attenuation.

About space charge injection of oil-paper sample, there are some differences in the total charge curves. As shown in Figure 14, in the short early stage of DC stressing (0 to $100 \mathrm{~s}$ ), the amount of space charge increased very fast, then it came to a temporary equilibrium state, after $20 \mathrm{~min}(1200 \mathrm{~s}) \mathrm{DC}$ stressing, the total charge amount decreased and then increased. A relative equilibrium of positive and negative charges appeared when the DC stressing continued.

However, in the volts-on tests, the variation curves of the total charge amount did not agreed very well. The main reason is that charge injection happened in a very short time, which would be effected by the environment conditions and the characteristics of oil-paper itself, such as ageing degree. Besides, in this paper, the time of volts-on test was basically within two hours, which mainly indicated the transfer and movement process of space charge, where a fluctuation in total charge amount was present. The charge equilibrium inside oil-paper may take a relatively longer time.

Therefore, a total charge accumulation pattern of oilpaper samples under different conditions is shown in Figure 15 . In the early short time, $0 \rightarrow t_{1}$ (less than $30 \mathrm{~s}$, may be a few seconds under some extreme conditions), it is from no space charge inside oil-paper to a certain amount injection inside. In the medium-term and anaphase, $t>t_{1}$, space charge transfers, gathers and neutralizes in oil-paper sample, from a long term and a whole point of view, total charge tends to be stable and balance.

The total charge accumulation pattern obeys the equation below

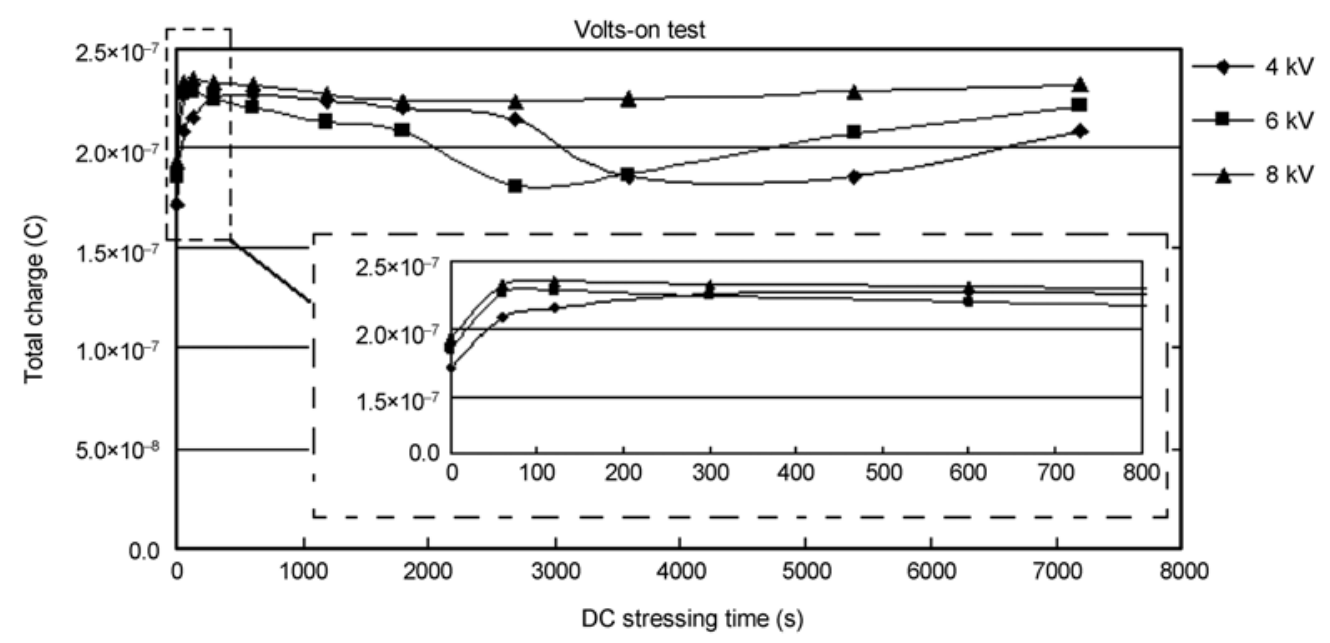

Figure 14 Total charge in the bulk vs. stressing time $\left(20^{\circ} \mathrm{C}\right)$. 


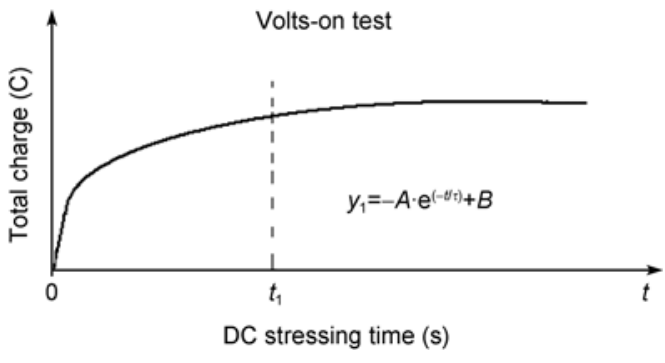

Figure 15 Total charge accumulation vs. stressing time.

$$
y_{1}=-A \cdot \mathrm{e}^{(-t / \tau)}+B .
$$

Compared with charge injection, the law of charge decay is quite evident (Figure 16). Once the applied voltage is moved, space charge trapped inside oil-paper moves towards the electrodes, in the early decay time (depends on the environment conditions and ageing degree of oil-paper, from a few minutes to dozens), $0 \rightarrow t_{1}$, the total charge amount decreases very fast at first then slows down. Subsequently, charge decays more slowly and finally goes to the ultimate state.

The total charge decay pattern obeys

$$
y_{2}=A \cdot \mathrm{e}^{(-t / \tau)}+B
$$

where $y_{1}$ and $y_{2}$ are the total charge of charge injection and decay; $A$ is the original amplitude constant, which indicates the initial value of total charge, as well as the initial trap energy distribution inside oil-paper; $B$ is the total amplitude constant, which indicates the value of total charge of oil-paper in the whole ageing process; $\tau$ is the speed constant, which indicates the speed of space charge increase and decrease; $t$ is the thermal ageing time.

From a large number of tests under different conditions, the injection and decay patterns of space charge in oil-paper insulation were proposed, and the physical meanings of the parameters in eqs. (12) and (13) were defined. In future work, based on the confirmation of the constants of a certain oil-paper material, along with some essential modifications of the equations, it is possible to realize the forecast and simulation of space charge behaviors inside oil-paper insulation.

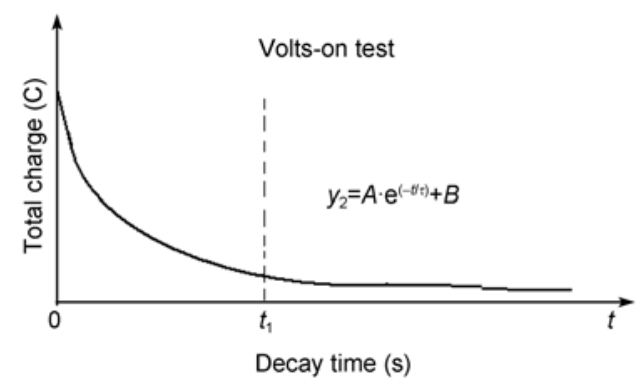

Figure 16 Total charge decay vs. stressing time.

\section{Conclusions}

In this paper, the PEA measurements on multi-layer oilimpregnated insulation paper samples were presented. Data mining and feature extractions were performed on the DC space charge behavior of oil-paper insulation. The conclusions are as follows.

1) Total charge: With the increase of DC stressing time, the total charge amount tended to stable after some fluctuation. The dissipation of total charge represents an exponential decay law.

2) The apparent charge mobility: On a whole the apparent charge mobility of oil-paper has a decreasing tendency with the increase of decay time. The present research indicates that test temperature and electrical field have different influences on the apparent charge mobility. At the same temperature, a higher electrical field will increase the apparent charge mobility. Under the same electrical field, the apparent charge mobility is relatively slow at a high temperature.

3) The trap energy distribution: Shallow traps which are much more than deep traps exist largely in the un-aged oil-paper. The effects and mechanisms of temperature and electrical field on the apparent charge mobility are basically the same as those on the trap depth. This paper established the relationship between depolarization and trap energy distribution, which realized the approximate calculation of trap energy in oil-paper through the decay test of space charge.

4) The accumulation and decay patterns of space charge in oil-paper insulation were acquired for the first time, and the physical meanings of the parameters in equations were defined, which will be helpful for forecast and simulation of space charge behaviors inside oil-paper insulation.

This work was supported by the National Basic Research Program of China ("973" Program) (Grant No. 2009CB724505-1), the Doctor Foundation of Southwest University (Grant No. SWU110038) and the Fundamental Research Funds for the Central Universities of Southwest University (Grant No. XDJK2010C020).

1 Boggs S. A rational consideration of space charge. IEEE Elect Insul Mag, 2004, 20: 22-27

2 Tanaka T, Greenwood A. Effects of charge injection and extraction on tree initiation in polyethylene. Power apparatus and systems. IEEE T Power Apparatus Syst, 1978, 5: 1749-1759

3 Maeda M, Tanaka A, Takahashi S, et al. Observation of charge behavior in organic photoconductor using pressure wave propagation method. In: Proceedings of the 3rd International Conference on Properties and Applications of Dielectric Materials. Tokyo: IEEE, 1991. 19: 1036-1039

4 Li Y, Murata K, Tanaka Y. Space charge distribution measurement in lossy dielectric materials by pulsed electroacoustic method. In: Proceedings of the 4th International Conference on Properties and Applications of Dielectric Materials. Brisbane: IEEE, 1994. 11: 725-728

5 Tanaka R, Sunaga H, Tamura N. The effect of accumulated charge on depth dose profile in poly (Methylmethacrylate) irradiated with fast electron beam. Nucl Sci, 1979, 26: 4670-4675 
6 Hozumi N, Tanaka J, Reggi A S, et al. Space charge induced in stressed polyethylene. In: IEEE Conference on Electrical Insulation and Dielectric Phenomena. Leesburg: IEEE, 1989. 12: 253-258

7 Mazzanti G, Montanari G C. Quantities extracted from space-charge measurements as markers for insulation aging. IEEE T Dielect El In, 2003, 10: 198-203

8 Montanari G C. Dielectric material properties investigated through space charge measurement. IEEE T Dielect El In, 2004, 11: 56-64

9 Dissado L, Mazzanti G, Montanari G C. A new thermo-electrical life model based on space-charge trapping. In: Conference Record of the 1996 IEEE International Symposium on Electrical Insulation. Quebec: IEEE, 1996. 16: 642-645

10 Liu F, Yang B, Tu D, et al. A new theory and experiment on electrical aging and breakdown of solid dielectrics. Acta Phys Sin, 1992, 21: 333-341

11 Yang B, Tu D, Liu Y. Dynamic process of the formation of ion space charge. J Xi'an Jiaotong Univ, 1991, 22: 89-96

12 Li J, Zhang Y, Zheng F, et al. The structure of XLPE and the distribution of space charge. Sci China Ser G-Phys Mech Astron, 2003, 46: 185-190

13 Zheng F, Zhang Y, Gong B, et al. Formation and migration of space charge packet in low density polyethylene. Sci China Ser E-Tech Sci, 2005, 48: 354-360

14 Yue X, Xia Z, Wang F. Study and development of polyethylene naphthalate. Sci China Ser E-Tech Sci, 2006, 9: 983-990

15 Li J, Zhang Y, Zheng F, et al. The structure configuration and space charge distribution in XLPE (in Chinese). Sci China Ser A-Math, 2002, 33: 1083-1087

16 Yang S, Xu Z, Wang Z, et al. Complex irradiancy of organic monolayer equipments under high voltage (in Chinese). Sci China Ser E-Tech Sci, 2002, 32: 191-197

17 Zhang W, Wang K, Liu Y, et al. Thermally stimulated current spectrum characteristics of nano-inorganic hybrided polyimide film. J Harbin Univ Sci Tech, 2004, 13: 40-42

18 Lu Y, Liang X, Zhou Y, et al. Study on space charge properties of DC composite insulator FRP rod materials. Insulating Mater, 2007, 40: 44-47

19 Zhou Y, Sun Q, Wang N, et al. Effects of space charges on DC breakdown in low density polyethylene. High Voltage Eng, 2008, 34: 26-31

20 Zhou Y, Wang Y, Wang N, et al. Effects of surface topography and morphology on space charge packet in polyethylene. High Voltage Eng, 2009, 35: 19-24

21 Liu R, Tornkvist C, Nsson K. Space charge distribution in composite oil cellulose insulation. In: IEEE International Conference on Dielectric Liquids. Coimbra, Portugal: IEEE, 2005. 10: 316-321

22 Liu R, Jaksts A. Moisture and space charge in oil-impregnated pressboard under HVDC. In: IEEE International Conference on Conduction and Breakdown in Solid Dielectrics. Sweden: IEEE, 1998. 21: 17-22

23 Morshuis P, Jeroense M. Space charge in HVDC cable insulation. In: IEEE Annual Report-Conference on Electrical Insulation and Dielectric Phenomena. Minneapolis: IEEE, 1997. 15: 28-32

24 Morshuis P, Jeroense M. Space charge measurements on impregnated paper a review of the PEA method and a discussion of results. IEEE Trans Electr Insul, 1997, 13: 26-35

25 Ciobanu R C, Pfeiffer W, Schreiner C. Charge packet evolution in paper-oil insulation and derived technological considerations. In: Proceedings of the 7th Intemational Conference on Properties and Applications of Dielectric Materials. Nagoya, Japan, 2003. 12: 695-698

26 Ciobanu R, Prisecaru I, Aradoaei S. PEA measurements upon cellulose materials submitted to gamma radiation. In: International Conference on Solid Dielectrics. France, 2004. 1: 225-228

27 Wang Y, Zhou Y, Li G, et al. Space charge phenomena in oil-paper insulation materials under high voltage direct current. High Voltage Eng, 2008, 25: 873-877

28 Chao T, George C, Ming F, et al. Space charge characteristics of multi-layer oil-paper insulation under different DC voltages. In: 9th International Conference on Properties and Applications of Dielectric Materials. Harbin, China, 2004. 5: 914-917

29 Tang C, Chen G, Fu M, et al. Effect of applied DC voltages and temperatures on space charge behavior of multi-layer oil-paper insulation. J Phys Conf Ser, 2009, 183: 775-784

30 Chao T, George C, Ming F, et al. Space charge behavior in multilayer oil-paper insulation under different DC voltages and temperatures. IEEE T Dielect El In, 2010, 17: 775-784

31 Wang X. Measuring energy distribution of surface trap in polymer insulation by PEA method. Proc CSEE, 2009, 29: 127-132

32 Chen G, Xiao H, Xia Z. Charge storage characteristics in hybrid electret film consisting of porous PTFE and PP with negative corona charging. Acta Phys Sin, 2006, 55: 2464-2469

33 Zhang Y, Yang B, Tu D. Measuring distribution of carrier trap energy state density at interface of dielectric with step pressure wave method. In: Conference on Electrical Insulation and Dielectric Phenomena. USA, 1989. 10: 303-308

34 Wang X. Analysis of finite element method for distortion of electrical stress by space charge in solid insulation materials. High Voltage Eng, 2001, 28: 51-53 